\title{
METHODS TO SUPPRESS POLARIZATION IN CHLORINE COMPENSATED CADMIUM TELLURIDE DETECTORS
}

\author{
P. SIFFERT, M. HAGE-ALI, R. STUCK and A. CORNET \\ Centre de Recherches Nucléaires, Laboratoire de Physique des Rayonnements \\ et d'Electronique Nucléaire, 67037 Strasbourg-Cedex, France
}

\begin{abstract}
Résumé. - Dans une première partie de ce travail on passe en revue les différents modèles de polarisation publiés dans la littérature. Puis, on étudie les caractéristiques essentielles du centre profond responsable de cet effet. Finalement, trois méthodes sont présentées qui permettent de s'affranchir de la polarisation : oxydation de la surface préalablement décapée chimiquement dans une solution de perhydrol puis dépôt de l'électrode conductrice, évaporation d'un film de $\mathrm{SiO}_{x}$, implantation ionique.
\end{abstract}

Abstract. - In a first part of this paper, the different models of polarization developped in the literature are critically analyzed. Then, the origin of the responsible center, its location within the bandgap and its concentration is investigated and discussed. Finally, three methods to suppress this effect are presented, mainly surface oxidation in perhydrol of the etched sample prior to the metal deposition, evaporation of a thin $\mathrm{SiO}_{x}$ layer or ion implantation.

Introduction. - It is well known that in nuclear radiation detectors prepared from insulating material appears a polarization effect, which is characterized by a progressive decrease of both pulse amplitude and counting rate with time after the bias voltage is switched on. This effect was responsible of the poor performance of the earliest (1945-50) solid state detectors (see references quoted in [1]). At that time, the exact mechanism was not completely understood and no effective procedure was found to overcome this effect. The development of the silicon junction type nuclear radiation detector considerably reduced the interest on insulating materials, only the diamond detectors continued to be investgated [2]. More recently, the possibility to use cadmium telluride in manufacturing room temperature $\gamma$-ray spectrometers has been considered and the travelling heater method (THM) has allowed the growth of high quality p-type crystals, in which the requested high resistivity $\left(\sim 10^{8} \Omega . \mathrm{cm}\right)$ is achieved by means of chlorine compensation. However, the nuclear radiation detectors prepared with this material show the typical decrease of pulse amplitude and counting rate with time. Several models have been proposed recently to explain the behaviour of $\mathrm{CdTe}(\mathrm{Cl})$ counters. These models will first be reviewed critically, then we will describe the three methods we developped to suppress polarization.

1. Models. - To explain the time dependent behaviour observed in these counters, it is necessary to consider, as done years ago for crystal counters by Hofstadter [3] that the electric field within the detector progressively changes, leading to the creation of a region of poor charge collection. Several groups $[4,1]$

have verified that in $\mathrm{CdTe}(\mathrm{Cl})$ detectors the initial constant field within the counter (identical to that existing in an n-i-p structure) progressively increases at the positive biased electrode and diminished at the opposite side, its shape becomes identical to that of an $\mathrm{n}-\mathrm{p}$ junction. This modification in the field distribution results from a change of the net charge carrier concentration (by trapping or detrapping) due to the progressive evolution of the occupancy of a deep level located either in the bulk or in close surface vicinity of the device.

2. Characteristics of the polarization level. - The main characteristics have been established by several groups, using quite different techniques, as shown on table I. However, the physical origin of the center is not clearly established today. Since polarization only occurs in THM grown crystals when chlorine is

\section{TABLE I}

\section{Characteristics of the deep level responsible for polarization}

$$
\begin{gathered}
\text { Energy location } \\
E_{\mathrm{T}}
\end{gathered}
$$

Ionized concentration

$$
N_{\mathrm{T}}\left(\mathrm{cm}^{-3}\right)
$$$$
\begin{aligned}
& E_{\mathrm{v}}+0.7 \mathrm{eV} \\
& E_{\mathrm{v}}+0.65(-0.70) \mathrm{eV} \\
& E_{\mathrm{v}}+0.85 \mathrm{eV}
\end{aligned}
$$

Ref.

[10]

$5 \times 10^{11}$

$5 \times 10^{10}$

$3 \times 10^{11}$

$10^{11}-10^{12}$

Capture cross-section $10^{-16}-10^{-17}$

$$
\sigma\left(\mathrm{cm}^{2}\right)
$$


present $[5,6]$, the simpliest hypothesis would be that this halogen introduces the deep level. In fact, chlorine introduces only a shallow level located at $E_{\mathrm{c}}-0.014 \mathrm{eV}$, its concentration in the crystals is generally close to $10^{17} \mathrm{~cm}^{-3}$. No study has been published until now about an eventual correlation between the concentration of chlorine and the amount of polarization, but luminescence measurements performed at $4.2 \mathrm{~K}$ indicated that the intensity of the line at $1.42 \mathrm{eV}$ (which corresponds to a level located at $E_{\mathrm{v}}+0.14 \mathrm{eV}$ ) is directly proportionnal to the importance of polarization [1]. This level, which is generally assumed to be due to the $\left[V_{\mathrm{cd}} \mathrm{Cl}\right]^{-}$complex is too shallow to be responsible of the effect, but its concentration is directly correlated to that of $\left[V_{\mathrm{cd}}\right]^{--}$, which introduces an acceptor level close to the midgap. The effect of chlorine introduction doping during the THM process is a strong enhancement of $\left[V_{\text {cd }}\right]^{--}$ from about $10^{7}$ to $10^{12} \mathrm{~cm}^{-3}$ as shown by our investigation of the compensation in CdTe [7]. Therefore, chlorine plays only an indirect role on polarization. The concentration of the midgap level becomes sufficient to contribute noticeably to the total charge concentration. Following this model, polarization does not just result from an increase in resistivity by compensation as often assumed.

3. How does this level become active. - The models proposed can be classified into two categories depending whenever they consider that polarization results from bulk or surface effects.

3.1 BuLK. - Following Malm et al. [4] when the bias voltage is switched on, the electric field sweeps the free carriers out of the interelectrode region, as in all detectors, causing a strong diminution of the charge carrier density. The deep acceptor level has to adjust slowly its ionization probability to correspond to the lower hole concentration, this is achieved by emission (detrapping) of holes which are bound to deep acceptors under zero field condition. Therefore, the polarization results from the time needed to achieve a new equilibrium. At equilibrium, the fraction $f$ of ionized deep acceptor is expressed by :

$$
f=\frac{1}{1+\sigma v p \tau_{\mathrm{D}}}
$$

where $\sigma v$ is the acceptor capture coefficient for holes, $p$ the concentration of holes and $\tau_{D}$ the detrapping time. The general expression of the ionization probability of a deep level located at $E_{\mathrm{T}}$, in a space charge region has been calculated by Shockley and Read [8] and is given by :

$$
\left\{1+\frac{C_{\mathrm{n}}}{C_{\mathrm{p}}} \frac{N_{\mathrm{c}}}{N_{\mathrm{v}}} \exp \left[-\frac{\left(E_{\mathrm{C}}-E_{\mathrm{T}}\right)+\left(E_{\mathrm{T}}-E_{\mathrm{v}}\right)}{k T}\right]\right\}^{-1}
$$

in which the symbols have their general meaning.
Malm et al. considered also the possibility that polarization may result from the capture (trapping) of free carriers, but they ruled out this hypothesis after they performed an experiment in which they show that the source strength has no influence on the amount of polarization. In a recent paper [1] we suggested that trapping can occur. Indeed, we demonstrated that the carriers participating to the polarization are those due to the space charge generated current rather than those created by the radiations, which are, generally, only a negligeable function of the total charge carriers, even with strong sources. For example, a $10 \mathrm{nA}$ leakage current corresponds approximatively to $10^{10}$ carriers/ sec and a source of $1 \mu \mathrm{C}$ strength of $100 \mathrm{keV}(4 \pi)$ generates less than $10^{9}$ carriers/s. Furthermore, the high activation energy may correspond to the barrier height of a negatively charged (repulsive) level to capture a second electron. This idea is confirmed by the fact that the capture cross section of the level $\left(\sim 10^{-17} \mathrm{~cm}^{2}\right.$ ) is close to that generally observed on repulsive centers.

It should be mentionned that this bulk model includes, in fact, strong surface effects, since the concentration of holes in relation (1) includes thoses injected through the positive biased contact. When $p$ is strongly enhanced (strong injection-low barrier height) the fraction of ionized deep acceptors diminishes and polarization vanishes, as experimentally verified $[9,1]$.

3.2 SuRfaCE. - Bell et al. [9] proposed that polarization results from the ionization of deep acceptors in the surface vicinity due to band bending, the level at $E_{\mathrm{T}}$ crossing the Fermi level. Due to the substantial activation energy which is required, the deep acceptor can only become ionized with a characteristic time constant. This model explains several observed effects but fails to show how the counting rate can become negligeable in some detectors. Under flat band conditions, no polarization would appear.

In our opinion [1] the major parameter in polarization is constituted by the charge injection through the positively biased electrode which modifies the location of the Fermi level. Since strong hole injection is not compatible with a low noise operation of the detector, solutions have to be found to control the rate of carrier injection.

4. Suppression of polarization. - The general idea in the research of methods to suppress polarization was to create a small concentration of electrons in the vicinity of the positively biased contact which is sufficient to recombine with the ionized acceptors but which does not affect the detectors operation. Bell et al. [10] have shown that shining strongly absorbed light on the positively biased contact creates sufficient electrons to overcome polarization. An ideal MIS structure on a P-type material, when positively biased would also create an excess electron density close to the contact. As shown on figure 1, when a small positive 

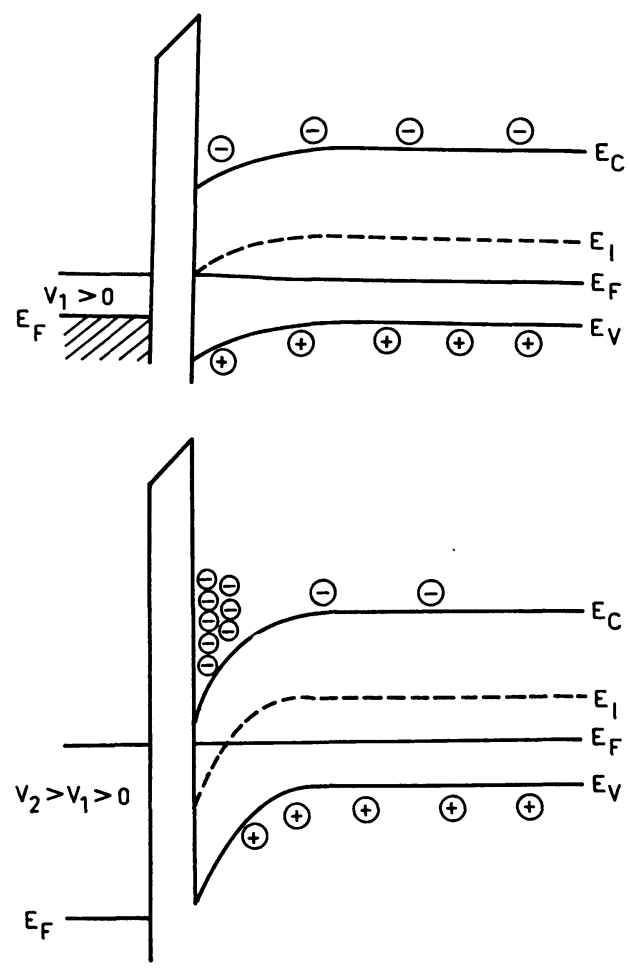

FIG. 1. - Reverse characteristic of both M-S and MIS CdTe detectors.

voltage is applied the bands bend downwards and the majority carriers are swept out of the zone (depletion structure). When the positive voltage is further increased, the bands bend even more, so that the intrinsic level $E_{\mathrm{i}}$ crosses the Fermi level, so the number of minority carriers (electrons) in the surface vicinity is larger than that of holes (inversion configuration). However, under ideal MIS conditions, no carrier flow is possible and the charges accumulated progressively diminish the electric field in the depletion zone, as in the structure developped by Eichinger [11, 12]. In a real integrated MIS structure some carrier flow is possible through the insulating layer. If the later is sufficiently thin, charge tunneling becomes possible. Therefore, we investigated the possibility to realize structures in which some current flow is possible. Three different methods have been considered on brominemethanol etched samples.

4.1 SILICON OXIDE LAYER. - Several methods have been proposed to deposit $\mathrm{SiO}_{x}$ films by vacuum evaporation of $\mathrm{SiO}$. The characteristics of the film strongly depend on the speed of evaporation, the residual pressure, the conditions of forming [13]. The exact mechanism of carrier transport through these films is not well known ; the simplest model [14] considers that some conductive filaments exist through the oxide layer through which some charge flow is possible.

Here, silicon monoxide was evaporated with an electron gun under a residual pressure of about $10^{-7}$ torr at moderate speed $(1 \AA / s)$ on the sample at thickness up to $100 \AA$. Under these experimental conditions, the oxide composition, as deduced from backscattering measurements, is about $\mathrm{SiO}_{1.8}$. The MIS structure is finally achieved by depositing either a gold or an aluminium electrode.

When used as nuclear detectors, voltages up to $700 \mathrm{~V}$ could be applied without any excess noise. However, the process is not yet fully controlled and it appears that more work is requested to fully control the charge flow through the oxide. Furthermore, the trapping center density at the oxide-semiconductor contact has to be carefully controlled to avoid any charge loss on these centers.

5. Boiling in hydrogen peroxide. - By boiling the etched samples during a few minutes in $\mathrm{H}_{2} \mathrm{O}_{2}$ an oxidized surface is formed, which is rich in cadmium, as shown by SIMS measurements. It is probable that a semiconductor layer similar to $\mathrm{CdO}$ is produced. The charge flow may occur either by hopping or Schottky emission over the interfacial layer. As shown on figure 2,

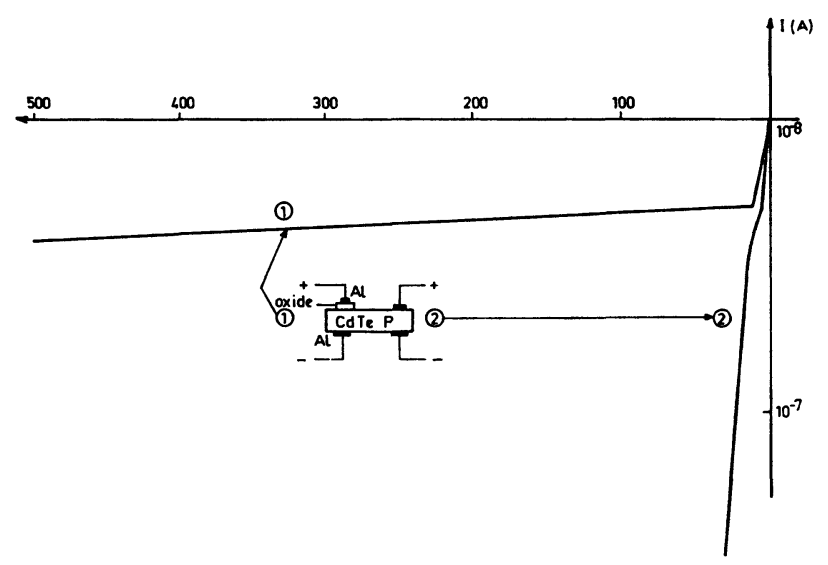

FIG. 2. - Energy band diagrams for ideal MIS structures at low $(a)$ and high $(b)$ bias voltage.

this procedure leads to a very strong reduction in diode leakage current and enhancement of the breakdown voltage.

5.1 Ion implantation. - The bombardment of compound semiconductor by heavy ions (or even protons) produces radiation damage centers which compensate the carriers existing previously in the material. Contrarily to the predictions of the theoretical models, the damage extends munch deeper into the material that the range of the implanted ions. Therefore, by a proper choice of dopant's energy and dosis it is possible to perform by ion implantation into CdTe both an insulating layer covered by a heavily doped surface film. It has been found that implantation of phosphorous, copper, gold or aluminium at energies ranging from 20 to $60 \mathrm{keV}$ and doses around $10^{14} \mathrm{~cm}^{-2}$ followed by an annealing around $200{ }^{\circ} \mathrm{C}$ gave the best results. 
6. Conclusion. - The results presented in this paper show that the problem of polarization on chlorine doped THM cadmium telluride crystals can be solved by a correct choice of the surface treatments. Therefore, it becomes now possible to realize small high performance nuclear counters on these materials. However, to fully use the possibilities of CdTe it is still necessary to improve the homogeneity of the THM crystals, in order to increase the active volumes.

\section{References}

[1] Siffert, P., Berger, R., Scharager, C., Cornet, A., Stuck, R., IEEE Trans. Nucl. Sci. NS 23 (1976) 159.

[2] Kozlov, S. R., Stuck, R., Hage-Ali, M., Siffert, P., IEEE Trans. Nucl. Sci. NS 22 (1975) 160.

[3] Hofstadter, R., Nucleonics 2, p. 29 (1949).

[4] Malm, H. L., Martini, M., Can. J. Phys. 51 (1973) 2336, id. IEEE Trans. Nucl. Sci. NS 21 (1974) 322.

[5] Triboulet, R., Cornet, A., Marfaing, Y., Siffert, P., J. Appl. Phys. 45 (1974) 2759, id. Nature Phys. Sci. 245 (1973) 12.

[6] Zanio, K., Krajenbrink, F., Montano, H., IEEE Trans. Nucl. Sci. NS 21 (1974) 315.

[7] Stuck, R., Cornet, A., Scharager, C., Siffert, P. J. Phys. Chem. Solids 37 (1976) 989.

[8] Shockley, W., Read, W. T., Phys. Rev. 87 (1952) 835.
[9] Bell, R. O., Entine, G., Serreze, H. B., Nucl. Instrum Meth. 117 (1974) 267.

[10] Bell, R. O., Wald, F. V., Goldner, R. B., IEEE Trans. Nucl. Sci. NS 22 (1975) 241.

[11] Eichinger, P., KallmanN, H., Z. Naturforsch 28a (1973) 1888, id. Appl. Phys. Lett. 25 (1974) 676.

[12] Eichinger, P., Halder, N., Kemmer, J., Nucl. Instrum. Meth. 117 (1974) 305.

[13] Howes, J. H., AwCock, M. L., Report Harwell AERER 6464 (1970).

[14] Dearnaley, G., Phys. Lett. 25A (1967) 760.

[15] Fabre, E., Ngo-Tich-Phuoc, Martin, G. M., Ortega. F., IEEE Trans. Nucl. Sci. NS 23 (1976) 182.

[16] Karpenko, V. P., Khasherininov, P. G., Matveev, O. A. Sov. Phys. Semicond. 4 (1971) 1492. 